\title{
Comprehensive Evaluation and Prediction Model of Sustainable Development
}

\author{
Jinlan Chen, Yuchen Xiao, Jialiang Zhou \\ School of Economics, Jinan University, Guangzhou, China \\ *chenjinlan_2006@foxmail.com
}

Keywords: comprehensive evaluation of sustainable development; sustainability prediction; BP Model; PCA; AHP

\begin{abstract}
The article conducts a research on sustainable development of a country or an area. We build a Short-term Evaluation Model and a Long-term Development to distinguish more sustainable countries from less sustainable ones, and combing the two models, we build comprehensive evaluation model of sustainable development. We select optimal plan and predict the sustainable development score in 20 years by AHP. Finally, BP Model is used to verify the efficiency of the prediction model.
\end{abstract}

\section{Introduction And Background}

How to manage increasing population and consumption with the earth's finite resources has been one of the largest challenges of our time. We are aim to decrease personal poverty and vulnerability, encourage economic development, and maintain ecosystem health.

\section{Comprehensive Evaluation Model of Sustainable Development}

Short-term Sustainable Evaluation Model. We use principal component analysis to measure three aspects index of social, economic development and ecosystem health. The evaluation of sustainable development is comprehensive evaluation of sustainable development usually divide the evaluated into several subsystems and come to a comprehensive system evaluation ${ }^{[1]}$.

- Select evaluation index. According to sustainable development indicator system(DSD) ${ }^{[2]}$. We search the information ${ }^{[3]}$ and get datum of 248 countries in social, economic development and ecosystem. So we select GDP, GDPPC and inflation rate as economic development index; Select health facility and total unemployment as social development index; Select flammable renewable resources, carbon dioxide emissions, and forestry area as ecosystem health index. The evaluation will be based on the 8 index.

- Data screening. We screen the countries with complete data from 1996 to 2010. There are 124 countries having been selected, such AGO, BHR and ZMB. We will use principal component analysis to the 124 countries.

- Compute and analysis. We analysis the data in 2006 using factor analysis, based on SPSS. We obtain 3 components, the eigenvalue of which is greater than 1 and the accumulating contribution rate of which reaches $70.017 \%$. So we select these 3 components as principal component to evaluate holistic data.

In addition, we also get the score of every component of every countries. The composite score of the sustainable evaluation is defined as

$$
\mathrm{y}_{i}=\sum_{j=1}^{3} w_{j} x_{i j}
$$

where ${ }^{y_{i}}$ is the composite scores of the ith country, ${ }^{w_{j}}$ is the contribution rate or weight of the ${ }^{j t h}$ component, ${ }^{x_{i j}}$ is the score of the ${ }^{j t h}$ component of the ith country.

Long-term Sustainable Evaluation Model. In Short-term Sustainable Evaluation Model, we can get score and degree of sustainable development of a country. However, only one year evaluation is 
not typical apparently. Sustainable development is a fairly long process, during which sustainability should be maintained.

\section{Two Vital Factors And Assumptions}

- Policy promoting: Positive policy is extremely crucial to sustainable development. Policy affects social, economic development and ecosystem health.

- Natural decay: We exactly know that there is a positive correlation between natural decay and the sustainable development level. In other words, more developed countries, because of driver of private interests, will decay faster without government management. Sustainable development level has some degree of decay.

Model construction. The changes of every year, comparing the last year, is decided on the two vital factors above, that is policy promoting and natural decay. We defined

$$
\Delta y=A-B
$$

where ${ }^{y}$ is the composite scores of a country; $A$ is the increment promoted by policy; $B=B(y)$ is the decrement by reason of natural decay, which is positive related to ${ }^{y}$.

We consider this situation is long-term and stable:

$$
\left\{\begin{array}{l}
\Delta y=0 \\
A=B \\
y=y_{\text {constant }}
\end{array}\right.
$$

A Scientific Definition of Sustainable Development. Combining Short-term Sustainable Evaluation Model and Long-term Sustainable Evaluation Model, we build a more comprehensive and scientific definition of sustainable development, shown in Table 1:

Table 1.

\begin{tabular}{c|c|c|c}
\hline $\begin{array}{c}\text { degree of sustainable } \\
\text { development in a long term }\end{array}$ & feeble sustainable & $\begin{array}{c}\text { moderate } \\
\text { sustainable }\end{array}$ & $\begin{array}{c}\text { strong } \\
\text { sustainable }\end{array}$ \\
\hline$y_{\text {constant }}$ & $>0.2$ & $>0.5$ & $>0.8$ \\
\hline
\end{tabular}

Creation of A 20-year Sustainable Development Plan. Combining the above-mentioned multi-aspect as consideration, we select Angola as the helped country to move towards a more sustainable future. The following will be further elaborated the evolution of long-term sustainable evaluation model, and create a 20 -year sustainable development plan.

The Long-term Evolution of Sustainable Development. In Long-term Sustainable Evaluation Model, we realize that the long-term evolution of sustainable development for its essence is the evolution process of the increment promoted by policy A between the decrement by reason of natural decay $\mathrm{B}$.

As shown in Figure 1, because $A$ is a constant, when $A>B$ as point $P$ shown, $\Delta y>0$, composite scores ${ }^{y}$ will increase and follow $B$ increasing, that is, $P$ is moving to the right to point $M \quad$ ( $A=B$, $\Delta y=0$ ). In this situation, the country reaches balance. Figure 2in Study of Coordinated Development Model and Its Application Between the Economy and Resources Environment in Small Town ${ }^{[4]}$, we find the The harmony coordinated point of regional development in the first quadrant proves the function of equantequation from another aspect, that is when $\alpha=45^{\circ}$, economic development and ecosystem health reaches balance and develop harmoniously. 


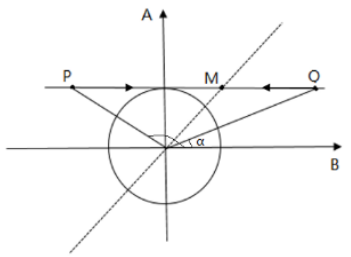

Figure 1.

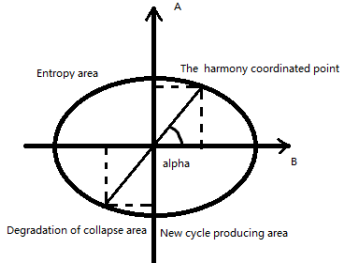

Figure 2.

\section{Future Prediction Model And Optimized Plans}

Construction of Future Prediction Model. On the basis on the 20-year sustainable development plan, we want to know the influence degree of the three parts of plans, program, policy and aid. So we use Analytic Hierarchy Process (AHP) so as to quantitative analysis to the sustainable development.

Calculating weight of the program, policy and aid, which is based on AHP.Let the goal be the score, the alternatives be the cost of the three parts of plans, program, policy and aid. We get three weight vectors. We select the cost of program, policy and aid as the additional environmental factors and add these factors into AHP. Then we can get different weights of three parts

$$
w_{i}=c_{1} a_{1 i}+c_{2} a_{2 i}+c_{3} a_{3 i}
$$

Throughout the different weights, we can find that, the three parts of plans, program, policy and aid effect the goal to various degree.

From Select evaluation index,we let the economic development index standardized value be $x_{11}, x_{12}$ and $x_{13}$; social development index standardized value be $x_{21}$ and $x_{22}$;ecosystem health index standardized value be $x_{31}, x_{32}$ and $x_{33}$.

- Calculating the increment promoted by policy $A$ :

Combining the weights, we can get the different increment promoted by different policy:

$$
\Delta x_{j k}=q w_{j} x_{j k}
$$

where ${ }^{w_{j}}$ is called as comprehensive influence coefficient in different parts of plans; ${ }^{q}$ is retardation coefficient. ${ }^{q}$ is negative related to ${ }^{y}$, which is defined as

$$
q=e^{-y}
$$

All $\Delta x_{j k}$ is substituted to Comprehensive Evaluation Model of Sustainable Development respectively and then use weighted summation. Eventually, we can get the increment promoted by policy $A$ contained by the increment of comprehensive score every year:

$$
A=A\left(\Delta x_{11}, \Delta x_{12}, \Delta x_{21}, \Delta x_{22}, \Delta x_{23}, \Delta x_{31}, \Delta x_{32}, \Delta x_{33}\right)
$$

- Calculating the decrement by reason of natural decay $B$ :

Because the decrement by reason of natural decay $B$ is positive related to ${ }^{y}$, we assume that $B=\delta y$

where $\delta$ is natural decay rate, ${ }^{y}$ is the composite scores of a country.

Add disturbance.Because of climate change, development aid, foreign investment, natural disasters, and government instability, the plans can not be carried out thoroughly and effectively, or there exist favorable opportunities to help development. Thus, we add some disturbance to the increment, a latest increment added disturbance is

$\Delta x_{j k}^{\prime}=\Delta x_{j k}(1+r)=w_{j} q_{j k} x_{j k}(1+r)$

where $r$ is a random number and $r \sim N(0,0.16)$.

\section{Model Results and Optimized Plans}

Model solving.We compare the effect on social, economic and ecosystem health respectively by program, policy and aid, and we can build the comparative matrix. We perform a consistency check and get the result:

$$
C R_{1}=-0.0901, C R_{2}=0.0462, C R_{3}=-0.0191 \text {. }
$$


We can find they are all less than 0.1. So we conclude the results pass the consistency check. According to the equation (4), we can get $w_{i}(i=1,2,3)$, s function expression. We standardize the data of 124 could in 2010, using SPSS. Equations $w_{i}(i=1,2,3)$ are substituted to Comprehensive Evaluation Model of Sustainable Development, taking retardation coefficient $q=e^{-y}=1.8$, and we will get the following equation:

$$
A=0.2437 * c_{1}+0.548 * c_{2}+0.2395 * c_{3}
$$

Additionally, we let the natural decay rate every year be $10 \%$, that is

$$
B=\delta y=0.1 y
$$

Optimized plans.Under the analysis above, we get the function of $A$ about $\left(c_{1}, c_{2}, c_{3}\right)$. Then if the cost of the plans is fixed, we can use MATLAB to find the optimized solution which maximize A. Here solve with nonlinear programming model as follow:

$$
A=0.2437 * c_{1}+0.548 * c_{2}+0.2395 * c_{3}
$$

S.t. $c_{1}^{2}+c_{2}^{2}+c_{3}^{2}=1, c_{1}, c_{2}, c_{3} \geq 0$

It is to be called the optimized plan which maximizes the policy promoting. We get the result: Program, policy and aid have the weights of 0.3794, 0.8522, 0.3604 .

Prediction and development with disturbance. We predict the sustainable development score in 20 years and Development with disturbance.

With an optimized plan given above, according to equation (10) and (11) and Long-term Evaluation Model, we can predict the composite sustainable scores in 20 years and draw trend chart as Figure 3.

On the basis of Future Prediction Model, add a disturbance quantity $r$, which follows Gaussian distributions with $\mu=0, \sigma=0.4$, that is $r \sim N(0,0.16)$. We compute stochastic disturbance process by MATLAB and draw Figure 4:

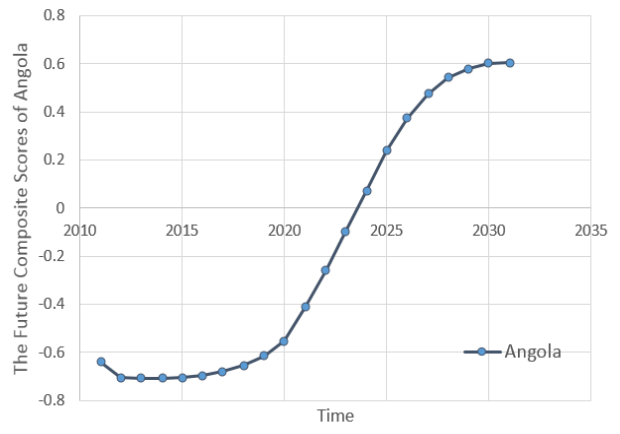

Figure 3.

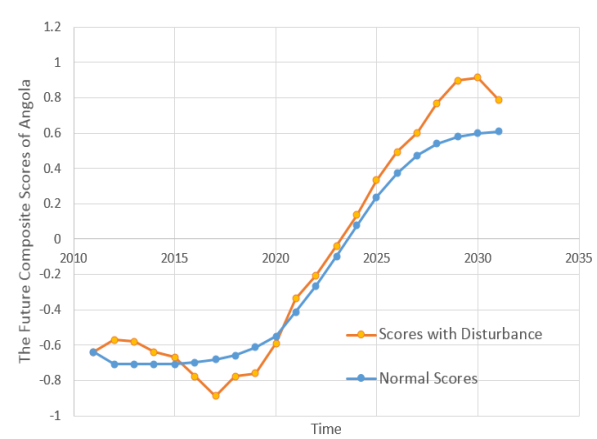

Figure 4.

Verification of The Plan Results.The evaluation model of sustainable development we explore is a complicated nonlinear system, so we use back propagation neural network (referred to as BP Algorithm) to predict the composite score of the sustainable development in a country. We can predict the sustainable development without plans through BP Model, which is compared with the plan results. Therefore we can explain how effective the plan is.

Construction of bp model.Back propagation neural network ${ }^{[5]}$ is multilayer feed-forward neural network of error back propagation. By training the sample data, the network weights and threshold value are modified, which leading to decreased error function along the negative gradient direction and close to the expected output. The topological structure of BP neural network consists of input layer, hidden layer and output layer. Hidden layer consists of one layer or several layers.

Model solution and realized.We set the neurons number of hidden layer be 6 ; the network epochs be 5000; the expected error be 0.00000001 ; learning rate be 0.01 . And the result is shown in Figure 5.below.

Figure 5 is the regression analysis of prediction values. Due to $\mathrm{R}>0.7$, we can safely drew a conclusion that the results fit well with historical data. Therefore data predicted by BP model could be regarded as a development without plans, while prediction in 4.2.3 is a development with plans. 
Comparing the prediction given above (the red line) with the plan results based on AHP from 4.2.3 (the black line), a trend chart could be drew by MATLAB as Figure 6:
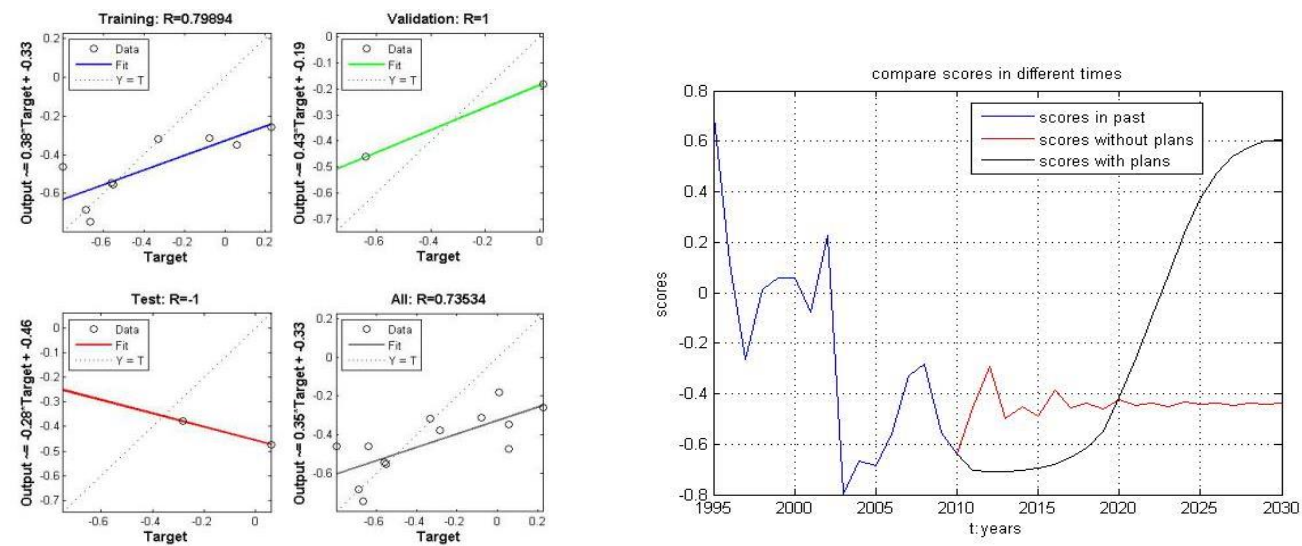

Figure 5.

Figure 6.

From the chart, it is obvious that development without plans (the red line) is more stable except a shock in the first few years, while the development with plans (the black line, data from 4.2.3) is going towards a high level until the score reach 0.6. So we can witness a great positive influence that the our plan could make on a country.

\section{References}

[1]Lee Song, He Jing. Research on Comprehensive Evaluation of Sustainable Development Based on Principal Component Analysis[A]. Journal of Liaoning Institutc of Technology.Volume 7, No.5, 2005 (Chinese)

[2]Evaluation Index System of Regional Sustainable Development, 07 Feb.2015, (Chinese)

[3]Information on http://data.worldbank.org

[4]Li Chongming,Ding Leiyun.Study of Coordinated Development Model and Its Application Betw een the Economy and Resources Environment in Small Town[A].Journal of Systems Science and In formation.No.11,2004

[5]BP neural network data forecast of the specific application and matlab code (Chinese) 